\title{
Treatment patterns and risk factor control in patients with and without metabolic syndrome in cardiac rehabilitation
}

This article was published in the following Dove Press journal:

Vascular Health and Risk Management

20 April 2012

Number of times this article has been viewed

\author{
Anselm Gitt ${ }^{\prime}$ \\ Christina Jannowitz ${ }^{2}$ \\ Marthin Karoff ${ }^{3}$ \\ Barbara Karmann ${ }^{2}$ \\ Martin Horack' \\ Heinz Völler ${ }^{4,5}$ \\ 'Institut für Herzinfarktforschung \\ an der Universität Heidelberg, \\ Ludwigshafen, ${ }^{2}$ Medical Affairs, MSD \\ Sharp and Dohme GmbH, Haar, \\ ${ }^{3}$ Klinik Königsfeld der Deutschen \\ Rentenversicherung Westfalen \\ in Ennepetal (NRW), Klinik der \\ Universität Witten-Herdecke, \\ ${ }^{4}$ Kardiologie, Klinik am See, \\ Rüdersdorf, ${ }^{5}$ Center of Rehabilitation \\ Research, University Potsdam, \\ Germany
}

Aim: Metabolic syndrome (MetS) is a clustering of factors that are associated with increased cardiovascular risk. We aimed to investigate the proportion of patients with MetS in patients undergoing cardiac rehabilitation (CR), and to describe differences between patients with MetS compared to those without MetS with regard to (1) patient characteristics including demographics, risk factors, and comorbidities, (2) risk factor management including drug treatment, and (3) control status of risk factors at entry to CR and discharge from CR.

Methods: Post-hoc analysis of data from 27,904 inpatients (Transparency Registry to Objectify Guideline-Oriented Risk Factor Management registry) that underwent a CR period of about 3 weeks were analyzed descriptively in total and compared by their MetS status.

Results: In the total cohort, mean age was 64.3 years, (71.7\% male), with no major differences between groups. Patients had been referred after a ST elevation of myocardial infarction event in $41.1 \%$ of cases, non-ST elevation of myocardial infarction in $21.8 \%$, or angina pectoris in $16.7 \%$. They had received a percutaneous coronary intervention in $55.1 \%$ and bypass surgery (coronary artery bypass graft) in $39.5 \%$. Patients with MetS $(n=15,819)$ compared to those without MetS $(n=12,085)$ were less frequently males, and in terms of cardiac interventions, more often received coronary artery bypass surgery. Overall, statin use increased from $79.9 \%$ at entry to $95.0 \%$ at discharge (MetS: $79.7 \%$ to $95.2 \%$ ). Patients with MetS compared to those without MetS received angiotensin converting enzyme inhibitors, angiotensin receptor blockers, oral antidiabetics, and insulin at entry and discharge more frequently, and less frequently clopidogrel and aspirin/clopidogrel combinations. Mean blood pressure was within the normal range at discharge, and did not differ substantially between groups (124/73 versus $120 / 72 \mathrm{mmHg}$ ). Overall, between entry and discharge, levels of total cholesterol, low density lipoprotein cholesterol, and triglycerides were substantially lowered, in particular in MetS patients. Thus, control rates of lipid parameters improved substantially, with the exception of high density lipoprotein cholesterol. Low density lipoprotein cholesterol rates $<100 \mathrm{mg} / \mathrm{dL}$ increased from $38.7 \%$ at entry to $73.8 \%$ at discharge (MetS: from $39.4 \%$ to $74.6 \%$ ) and triglycerides control rates $(<150 \mathrm{mg} / \mathrm{dL}$ ) from $58.1 \%$ to $70.4 \%$ (MetS: $43.7 \%$ to $62.2 \%$ ). Physical fitness on exercise testing improved substantially in both groups.

Conclusion: Patients with and without MetS benefited substantially from the participation in CR, as their lipid profile, blood pressure, and physical fitness improved. Treatment effects were similar in the two groups.

Keywords: cardiac rehabilitation, registry, metabolism, diabetes, dyslipidemia, control rates, risk factor, lipids

\section{Introduction}

Despite a declining incidence in recent years, cardiovascular events remain a leading cause of morbidity and mortality in most regions worldwide. In clinical practice,
Correspondence: Heinz Völler

Klinik am See, Seebad 84,

D-15562 Rüdersdorf, Germany

Tel +493363878 623

$\mathrm{Fax}+493363878624$

Email heinz.voeller@klinikamsee.com 
the prediction of such events to identify patients at high cardiovascular risk and to offer them preventive measures is of high priority. ${ }^{1}$ Substantial research has been performed on the clustering of cardiovascular risk factors named metabolic syndrome (MetS) and the question whether this clustering represents a disease entity in its own right. At least five definitions with slight or moderate deviations from each other have been proposed by international organizations or by expert groups..$^{2-7}$ The most frequently used ones have been issued by the National Cholesterol Education Program Adult Treatment Panel (NCEP ATP) III $^{5}$ and by the International Diabetes Federation (IDF). ${ }^{7}$

A setting with a known high prevalence of patients with MetS is cardiac rehabilitation (CR). CR is primarily indicated for patients who have received a diagnosis of acute myocardial infarction, have undergone coronary revascularization, or have chronic stable angina. ${ }^{8,9}$ The goals of $\mathrm{CR}$ and secondary prevention involve a program of prescribed exercise and interventions designed to modify coronary risk factors, including drug therapy. The aims are to prevent disability resulting from coronary disease, particularly in elderly persons and those with occupations that involve physical exertion, and to prevent subsequent coronary events, subsequent hospitalization, and death from cardiac causes. ${ }^{8}$ The benefits of CR are broad and compelling. Exercise together with nutritional counseling can slow the atherosclerotic process ${ }^{10,11}$ and lead to lower rates of cardiac events and hospitalizations. ${ }^{10,12}$ In a review of 22 studies, an average mortality reduction of $20 \%$ has been described for patients who underwent $\mathrm{CR}$ with exercise. ${ }^{13}$

In Germany, the transfer of cardiac patients from the hospital to the CR clinic is an established procedure with low barriers, and is particularly independent of socioeconomic status. Thus the situation represents not usual but rather best practice, which can be analyzed to investigate effectiveness of therapy under real practice conditions. ${ }^{9,14}$

It can be expected that a higher percentage of cardiac patients in CR fulfill the definition of MetS, but to date no specific data have been reported in this setting. The Transparency Registry to Objectify Guideline-Oriented Risk Factor Management (TROL) is one of the largest registries on $\mathrm{CR}$ and has been used, among others, for the description of secular trends in the management of patients in "realworld" CR. ${ }^{15}$ We performed a post-hoc analysis of data of the registry with the aim to investigate the proportion of patients with MetS in patients undergoing CR, and to describe differences between patients with MetS compared to those without MetS using the IDF definition ${ }^{7}$ with regard to (1) patient characteristics including demographics, risk factors, and comorbidities, (2) risk factor management including drug treatment, and (3) control status of risk factors at entry to $\mathrm{CR}$ and discharge from $\mathrm{CR}$.

\section{Methods \\ Source of data}

TROL is a large scale prospective registry set up in 2003 under the auspices of the German Society for Prevention and Rehabilitation. ${ }^{16}$ Centers were eligible to participate if they had a significant number of CR patients, and were selected across Germany to ensure that all 16 federal states were represented adequately. ${ }^{15,17}$ Participating physicians documented inpatients on standardized case report forms. The ethics committee of the Bavarian Physician Chamber approved the study, and all patients provided informed consent. Patient data protection was ensured. We report an analysis of the dataset of the years 2005 to 2008, which comprises 27,904 patients with adequate and complete information to decide on the presence of MetS.

\section{CR program}

CR programs are not standardized in Germany in terms of type (eg, inpatient versus outpatient), length, structure, or components. However, the vast majority of patients undergo in-hospital rehabilitation therapy usually for 3-4 weeks. ${ }^{9}$ Key features of these programs are gradual increase in activity levels, continuation of risk factor modifications (eg, lipid lowering), and development of maintenance programs. ${ }^{9,15}$

\section{Variables}

The following patient characteristics were recorded: (1) demographics: age, gender, body mass index (BMI); (2) length of stay: rehabilitation beginning and end; (3) risk factors (diabetes mellitus, hyperlipoproteinemia, arterial hypertension, smoking, positive cardiac family history); (4) concomitant diseases: peripheral arterial disease, previous stroke; (5) systolic and diastolic blood pressure at entry and discharge; (6) laboratory parameters at entry and discharge: total cholesterol ( $\mathrm{TC}, \mathrm{mg} / \mathrm{dL})$, low density lipoprotein cholesterol (LDL-C, mg/dL), high density lipoprotein cholesterol (HDL-C, mg/dL), triglycerides (TG, mg/dL), fasting blood glucose $(\mathrm{mg} / \mathrm{dL}) ;(7)$ cardiopulmonary exercise testing (Watts); (8) medication use at entry and discharge: statins, acetylic salicylic acid (ASA, aspirin), beta blockers, angiotensin converting enzyme (ACE) inhibitors, angiotensin receptor antagonists (ARB), oral antidiabetic drugs, insulin, and other drugs. 
Patients were categorized as having MetS if they met the definition of the IDF $2005^{7}$ (Table 1).

\section{Statistical analysis}

Data are presented as absolute numbers, percentages, or means with standard deviations (SD). The frequencies of categorical variables in populations were compared by $\mathrm{Chi}$ square test. Continuous variables were compared by twotailed Wilcoxon rank sum test. Percentages were calculated on the basis of patients with data for each respective parameter (ie, no percentages for missing values provided). The analysis was performed with the SAS (v9.1; SAS Institute Inc, Cary, NC).

\section{Results}

\section{Patient demographics and characteristics} Total cohort

A total of 27,904 patients with information on MetS (15,819 with MetS/12,085 without MetS) were available (in 2005: 5805; 2006: 8839; 2007: 5753; 2008: 7507 patients). The majority of patients were fully or partly institutionalized for CR (95.3\% or $1.4 \%$, respectively). Mean duration of rehabilitation was $21.4 \pm 37.5$ days, with a trend towards an increase over the years (2005: 19.8 days; 2006: 20.3 days; 2007: 22.9 days; 2008: 22.7 days). The majority of patients were retired $(61.5 \%)$.

Table I IDF definition of MetS $\mathrm{S}^{7,40}$

\begin{tabular}{|c|c|}
\hline Parameter & Explanation \\
\hline Central obesity & $\begin{array}{l}\text { Defined as waist circumference } \geq 94 \mathrm{~cm} \text { for } \\
\text { European men and } \geq 80 \mathrm{~cm} \text { for European } \\
\text { women, with ethnicity specific values for other } \\
\text { groups* }\end{array}$ \\
\hline $\begin{array}{l}\text { plus any two of the } \\
\text { following four factors: }\end{array}$ & \\
\hline Raised TG & $\begin{array}{l}\geq 1.7 \mathrm{mmol} / \mathrm{L}(150 \mathrm{mg} / \mathrm{dL}) \\
\text { or specific treatment for this lipid abnormality }\end{array}$ \\
\hline Reduced HDL-C & $\begin{array}{l}<1.03 \mathrm{mmol} / \mathrm{L}(40 \mathrm{mg} / \mathrm{dL}) \text { in males } \\
<1.29 \mathrm{mmol} / \mathrm{L}(50 \mathrm{mg} / \mathrm{dL}) \text { in females } \\
\text { or specific treatment for this lipid abnormality }\end{array}$ \\
\hline Raised blood pressure & $\begin{array}{l}\text { Systolic: } \geq 130 \mathrm{mmHg} \text { or diastolic: } \geq 85 \mathrm{mmHg} \\
\text { or treatment of previously diagnosed } \\
\text { hypertension }\end{array}$ \\
\hline $\begin{array}{l}\text { Raised plasma } \\
\text { glucose** }\end{array}$ & $\begin{array}{l}\text { Fasting plasma glucose } \geq 5.6 \mathrm{mmol} / \mathrm{L}(100 \mathrm{mg} / \mathrm{dL}) \\
\text { or previously diagnosed type } 2 \text { diabetes }\end{array}$ \\
\hline
\end{tabular}

Notes: *If BMI is $>30 \mathrm{~kg} / \mathrm{m}^{2}$, central obesity can be assumed and waist circumference does not need to be measured; **In clinical practice, impaired glucose tolerance is also acceptable, but all epidemiological reports of the prevalence of MetS should use only fasting plasma glucose and presence of previously diagnosed diabetes to assess this criterion. Prevalence also incorporating 2-hour glucose results can be added as supplementary findings.

Abbreviations: IDF, International Diabetes Federation; MetS, metabolic syndrome; TG, triglycerides; HDL-C, high density lipoprotein cholesterol; BMI, body mass index.
Demographic and clinical characteristics are summarized in Table 2. The proportion of males in total was $71.7 \%$, the average age $64.3 \pm 11.5$ years, the mean BMI was $28.4 \pm 4.8 \mathrm{~kg} / \mathrm{m}^{2}$. Cardiovascular risk factors were highly prevalent as expected, in particular lipid disorders (97.1\%), diabetes mellitus (35.7\%), arterial hypertension (86.9\%), and former or current smoking ( $47.7 \%$ and $15.7 \%$, respectively). In addition to coronary artery disease, $12.2 \%$ of patients also had peripheral artery disease, and $8.8 \%$ had had a prior stroke event.

Patients with ST elevation myocardial infarction (STEMI) were the largest group ( $41.1 \%$ of patients), followed by nonST elevation myocardial infarction (NSTEMI; 21.8\%) and instable angina pectoris $(16.7 \%)$. In terms of therapy at the acute hospital, percutaneous coronary intervention was more often reported compared to coronary artery bypass graft (55.1\% versus $39.5 \%$, respectively).

\section{Subgroups with and without MetS}

Due to the large sample size, statistically significant differences between the two subgroups were noted for all mentioned demographic and clinical characteristics with the exception of NSTEMI. Patients with MetS compared to those without MetS were less frequently men, and naturally differed with respect to components of the MetS definition (having a higher BMI, a higher waist circumference, more often hypertension, and substantially more often diabetes ( $52 \%$ versus $14 \%$, respectively). Mean fasting glucose was higher in patients with MetS compared to those without MetS (115 mg/dL versus $98 \mathrm{mg} / \mathrm{dL})$, as was glycosylated hemoglobin $\left(\mathrm{HbA}_{1 \mathrm{c}} ; 6.6 \%\right.$ versus $\left.6.1 \%\right)$. In terms of cardiac interventions, patients with MetS more often received coronary artery bypass graft surgery.

\section{Drug utilization}

\section{Total cohort}

Drug treatment at entry and discharge is shown in Table 3. In the total cohort, the majority of patients received statins at entry (any drug in $79.9 \%)$. In particular, simvastatin (61.0\%, at a mean dose of $30.4 \mathrm{mg} /$ day), atorvastatin ( $7.0 \%$, mean dose $28.9 \mathrm{mg} /$ day), and fluvastatin ( $7.9 \%$, mean dose $60.2 \mathrm{mg} /$ day) were reported. At the end of CR stay, almost all patients received statin therapy (any statin in 95.2\%). The rates of simvastatin use increased, while all other statins decreased somewhat. Furthermore, overall the respective mean dosages increased slightly, eg, for atorvastatin to $32.3 \mathrm{mg} / \mathrm{day}$.

Treatment with cholesterol absorption inhibitors increased substantially during the CR (from $7.2 \%$ to $47.2 \%$ ). 
Table 2 Demographic and clinical factors in patients with and without MetS

\begin{tabular}{|c|c|c|c|c|c|}
\hline Parameter & $\begin{array}{l}\text { Total } \\
n=27,904\end{array}$ & $\begin{array}{l}\text { MetS } \\
n=15,819\end{array}$ & $\begin{array}{l}\text { No MetS } \\
n=12,085\end{array}$ & $P$ value* & Odds ratio* \\
\hline \multicolumn{6}{|l|}{ Demographics } \\
\hline Age, years & $64.3 \pm 11.5$ & $64.6 \pm 11.2$ & $63.9 \pm 11.8$ & $<0.0001$ & \\
\hline Gender, male, \% & 71.7 & 67.6 & 77.2 & $<0.0001$ & 0.62 \\
\hline Weight, kg & $82.4 \pm 16.1$ & $87.0 \pm 16.2$ & $76.4 \pm 13.8$ & $<0.0001$ & \\
\hline Body mass index, $\mathrm{kg} / \mathrm{m}^{2}$ & $28.37 \pm 4.78$ & $30.0 \pm 4.71$ & $26.24 \pm 3.95$ & $<0.000$ I & \\
\hline Waist circumference, $\mathrm{cm}$ & $99.5 \pm 13.5$ & $105.0 \pm 11.8$ & $92.5 \pm 12.2$ & $<0.0001$ & \\
\hline \multicolumn{6}{|l|}{ Diagnosis for CR } \\
\hline STEMI, \% & $4 I .1$ & 38.7 & 44.3 & $<0.0001$ & $0.79(0.76-0.83)$ \\
\hline NSTEMI, \% & 21.8 & 21.7 & 21.9 & 0.72 & $0.99(0.93-1.05)$ \\
\hline Unstable angina pectoris, \% & 16.7 & 17.3 & 15.9 & $<0.01$ & $1.10(1.04-1.18)$ \\
\hline \multicolumn{6}{|l|}{ Therapy in acute hospital } \\
\hline $\mathrm{PCl}, \%$ & 55.1 & 52.7 & 58.3 & $<0.0001$ & $0.80(0.76-0.84)$ \\
\hline Coronary artery bypass, $\%$ & 39.5 & 42.2 & 35.9 & $<0.0001$ & $1.30(1.24-1.37)$ \\
\hline \multicolumn{6}{|l|}{ Risk factors } \\
\hline Diabetes mellitus, \% & 35.7 & 52.1 & 14.0 & $<0.0001$ & $6.66(6.26-7.08)$ \\
\hline Dyslipidemia, \% & 97.1 & 97.7 & 96.3 & $<0.000 \mathrm{I}$ & $1.63(1.42-1.88)$ \\
\hline Arterial hypertension, \% & 86.9 & 94.6 & 76.8 & $<0.0001$ & $5.26(4.85-5.70)$ \\
\hline Smoking, current, \% & 15.7 & 14.4 & 17.5 & $<0.000 \mathrm{I}$ & $0.79(0.74-0.85)$ \\
\hline Smoking, previous, \% & 47.7 & 48.0 & 47.2 & 0.23 & $1.03(0.98-1.08)$ \\
\hline Peripheral arterial disease, $\%$ & 12.2 & 12.9 & 11.3 & $<0.0001$ & $1.16(1.08-1.25)$ \\
\hline Previous stroke, $\%$ & 8.8 & 9.4 & 8.0 & $<0.000 \mathrm{I}$ & $1.19(1.10-1.30)$ \\
\hline Positive family history, $\%$ & 30.5 & 31.3 & 29.5 & 0.01 & $1.09(1.03-1.15)$ \\
\hline
\end{tabular}

Note: ${ }^{*} P$ values and odds ratios refer to the comparison between the two groups (MetS versus no MetS) at entry.

Abbreviations: MetS, metabolic syndrome; CR, cardiac rehabilitation; STEMI, ST elevation myocardial infarction; NSTEMI, non-ST elevation myocardial infarction; PCl, percutaneous coronary intervention.

ASA use remained nearly unchanged at a high level (at discharge $84.2 \%$ ), while clopidogrel alone or in combination with ASA decreased somewhat. ACE inhibitors and ARBs were frequently used in this study (at discharge $72.6 \%$ and $16.3 \%$, respectively).

\section{Subgroups with and without MetS}

In the statistical comparison, there were statistically significant differences for almost all drug classes between patients without MetS and those with MetS. To note major differences, patients with MetS had more frequent use of

Table 3 Drug treatment at entry and discharge

\begin{tabular}{|c|c|c|c|c|c|}
\hline Parameter & $\begin{array}{l}\text { Total } \\
n=27,904\end{array}$ & $\begin{array}{l}\text { MetS } \\
n=15,819\end{array}$ & $\begin{array}{l}\text { No MetS } \\
n=12,085\end{array}$ & $P$ value* & Odds ratio* \\
\hline Statins, any \% & $79.9 \rightarrow 95.2$ & $79.7 \rightarrow 95.2$ & $80.2 \rightarrow 95.4$ & $0.30 \rightarrow 0.40$ & $0.97 \rightarrow 0.95$ \\
\hline Simvastatin, \% & $61.0 \rightarrow 83.2$ & $60.6 \rightarrow 82.7$ & $61.6 \rightarrow 83.8$ & $0.08 \rightarrow<0.05$ & $0.96 \rightarrow 0.92$ \\
\hline \multirow[t]{2}{*}{ Dose, mg/day $\pm S D$} & $30.4 \pm 12.7 \rightarrow$ & $30.5 \pm 12.7 \rightarrow$ & $30.3 \pm 12.6 \rightarrow$ & $0.34 \rightarrow 0.4 \mathrm{I}$ & \\
\hline & $31.3 \pm 13.9$ & $31.3 \pm 13.9$ & $31.4 \pm 13.9$ & & \\
\hline Pravastatin, \% & $3.8 \rightarrow 2.0$ & $3.8 \rightarrow 2.0$ & $3.8 \rightarrow 2.0$ & $0.97 \rightarrow 0.85$ & $1.00 \rightarrow 1.02$ \\
\hline Atorvastatin, \% & $7.0 \rightarrow 4.4$ & $7.1 \rightarrow 4.5$ & $6.7 \rightarrow 4.2$ & $0.22 \rightarrow 0.37$ & $1.06 \rightarrow 1.05$ \\
\hline Fluvastatin, \% & $7.9 \rightarrow 5.5$ & $8.0 \rightarrow 5.7$ & $7.8 \rightarrow 5.2$ & $0.69 \rightarrow<0.05$ & $1.02 \rightarrow 1.11$ \\
\hline Other statin, \% & $0.5 \rightarrow \mathrm{I} .8$ & $0.5 \rightarrow \mathrm{I} .8$ & $0.4 \rightarrow \mathrm{I} .8$ & $0.24 \rightarrow 0.86$ & $1.23 \rightarrow 0.98$ \\
\hline $\mathrm{CAl}, \%$ & $7.2 \rightarrow 47.2$ & $7.6 \rightarrow 47.0$ & $6.7 \rightarrow 47.3$ & $<0.01 \rightarrow 0.65$ & $1.14 \rightarrow 0.99$ \\
\hline ASA, $\%$ & $83.8 \rightarrow 84.2$ & $83.0 \rightarrow 83.4$ & $84.8 \rightarrow 85.2$ & $<0.000 \mathrm{I} \rightarrow<0.000 \mathrm{I}$ & $0.88 \rightarrow 0.87$ \\
\hline ASA + clopidogrel, $\%$ & $45.2 \rightarrow 42.0$ & $42.2 \rightarrow 39.0$ & $49.2 \rightarrow 46.0$ & $<0.000 \mathrm{I} \rightarrow<0.000 \mathrm{I}$ & $0.76 \rightarrow 0.75$ \\
\hline Beta blocker, \% & $86.2 \rightarrow 89.5$ & $87.1 \rightarrow 90.3$ & $85.0 \rightarrow 88.6$ & $<0.000 \mathrm{I} \rightarrow<0.000 \mathrm{I}$ & $1.19 \rightarrow 1.20$ \\
\hline ACE inhibitor, \% & $71.5 \rightarrow 72.6$ & $72.0 \rightarrow 73.3$ & $70.9 \rightarrow 71.6$ & $<0.05 \rightarrow<0.01$ & $1.05 \rightarrow 1.09$ \\
\hline $\mathrm{ARB}, \%$ & $12.4 \rightarrow 16.3$ & $14.0 \rightarrow 18.4$ & $10.4 \rightarrow 13.6$ & $<0.000 \mathrm{I} \rightarrow<0.000 \mathrm{I}$ & $\mathrm{I} .4 \mathrm{I} \rightarrow \mathrm{I} .44$ \\
\hline Oral antidiabetic drug, \% & $15.3 \rightarrow 16.9$ & $22.9 \rightarrow 25.3$ & $5.3 \rightarrow 6.1$ & $<0.000 \mathrm{I} \rightarrow<0.000 \mathrm{I}$ & $5.3 \rightarrow 5.23$ \\
\hline Insulin, \% & $12.3 \rightarrow 12.6$ & $18.1 \rightarrow 18.5$ & $4.7 \rightarrow 4.8$ & $<0.000 \mathrm{I} \rightarrow<0.000 \mathrm{I}$ & $4.49 \rightarrow 4.54$ \\
\hline
\end{tabular}

Notes: $* P$ values and odds ratios refer to the comparison between the two groups (MetS versus no MetS) at entry. Values are percentages at entry and $\rightarrow$ at discharge. Abbreviations: MetS, metabolic syndrome; SD, standard deviation; CAI, cholesterol absorption inhibitor; ASA, acetylic salicylic acid; ACE, angiotensin converting enzyme; ARB, angiotensin receptor blocker. 
ACE inhibitors and ARBs (at entry and discharge), and less frequent use of clopidogrel and ASA/clopidogrel combinations. With respect to antidiabetic medications, patients with MetS had higher use of oral antidiabetic drugs $(22.9 \%$ versus $5.3 \%$ at entry and $25.3 \%$ versus $6.1 \%$ at discharge) and insulin (18.1\% versus $4.7 \%$ at entry and $18.5 \%$ versus $4.8 \%$ at discharge).

\section{Target level attainment}

\section{Total cohort}

Lipid levels, other surrogate parameters, and target level attainment at entry and at discharge are shown in Table 4. In the total cohort at entry mean TC was $184.9 \mathrm{mg} / \mathrm{dL}$, mean LDL-C $113.8 \mathrm{mg} / \mathrm{dL}$, mean HDL-C $43.2 \mathrm{mg} / \mathrm{dL}$, and mean TG $157.4 \mathrm{mg} / \mathrm{dL}$.

At discharge, mean values had considerably improved (TC $152.6 \mathrm{mg} / \mathrm{dL}$, LDL-C $86.1 \mathrm{mg} / \mathrm{dL}$, and TC $136.1 \mathrm{mg} / \mathrm{dL}$ ), while HDL-C remained unchanged.
Figure 1A-E display control rates of various lipid parameters alone and in combination at entry and discharge, in the total cohort and by subgroup. Overall, between entry and discharge, control rates of lipid parameters improved substantially, with the exception of HDL-C. The LDL-C goal $(<100 \mathrm{mg} / \mathrm{dL})$ was achieved at entry by $38.7 \%$ of patients and at discharge by $73.8 \%$, the TC goal $(<200 \mathrm{mg} / \mathrm{dL})$ at entry by $66.7 \%$ of patients and at discharge by $90.3 \%$, the HDL-C goal ( $>50 \mathrm{mg} / \mathrm{dL}$ in women; $>40 \mathrm{mg} / \mathrm{dL}$ in men) at entry by $44.0 \%$ of patients and at discharge by $44.4 \%$. Furthermore, the TG goal of $<150 \mathrm{mg} / \mathrm{dL}$ was met at entry by $58.1 \%$ of patients and at discharge by $70.4 \%$. Combined lipid goals for HDL-C + TG were reached by $29.0 \%$ of patients at entry and by $34.6 \%$ at discharge; for LDL-C + HDL-C + TG by $11.5 \%$ at entry and by $25.9 \%$ at discharge.

Mean systolic and diastolic blood pressure were decreased to $122 / 72 \mathrm{mmHg}$ (entry: $130 / 77 \mathrm{mmHg}$ ), and the maximum exercise capacity increased to 94 Watts (entry: 77 Watts).

Table 4 Parameters and treatment goal achievement at entry and discharge

\begin{tabular}{|c|c|c|c|c|c|}
\hline Parameter & $\begin{array}{l}\text { Total } \\
\mathrm{n}=\mathbf{2 7 , 9 0 4}\end{array}$ & $\begin{array}{l}\text { MetS } \\
n=\mid 5,819\end{array}$ & $\begin{array}{l}\text { No MetS } \\
n=12,085\end{array}$ & $P$ value* & Odds ratio* \\
\hline $\mathrm{TC}(\mathrm{mg} / \mathrm{dL})$ & $\begin{array}{l}\mid 84.9 \pm 48.0 \rightarrow \\
|52.6 \pm 36 .|\end{array}$ & $\begin{array}{l}184.8 \pm 49.6 \rightarrow \\
151.0 \pm 37.0\end{array}$ & $\begin{array}{l}|85 .| \pm 45.3 \rightarrow \\
\mid 55.0 \pm 35.2\end{array}$ & $<0.05 \rightarrow<0.0001$ & NA \\
\hline LDL-C (mg/dL) & $\begin{array}{l}\mid 13.8 \pm 39.1 \rightarrow \\
86.1 \pm 28.6\end{array}$ & $\begin{array}{l}113.9 \pm 40.1 \rightarrow \\
85.4 \pm 28.9\end{array}$ & $\begin{array}{l}I 13.7 \pm 37.6 \rightarrow \\
87.1 \pm 28.0\end{array}$ & $0.26 \rightarrow<0.000 \mathrm{I}$ & NA \\
\hline HDL-C (mg/dL) & $\begin{array}{l}43.2 \pm 12.8 \rightarrow \\
43.2 \pm 12.0\end{array}$ & $\begin{array}{l}39.9 \pm 11.1 \rightarrow \\
40.2 \pm 10.6\end{array}$ & $\begin{array}{l}48.2 \pm 13.6 \rightarrow \\
47.7 \pm 12.7\end{array}$ & $<0.000 \mathrm{I} \rightarrow<0.000 \mathrm{I}$ & NA \\
\hline TG (mg/dL) & $\begin{array}{l}\mid 57.4 \pm 81.0 \rightarrow \\
|36.1 \pm 7| .3\end{array}$ & $\begin{array}{l}|78 .| \pm 94.5 \rightarrow \\
|49.8 \pm 76 .|\end{array}$ & $\begin{array}{l}126.0 \pm 62.2 \rightarrow \\
115.3 \pm 57.4\end{array}$ & $<0.000 \mathrm{I} \rightarrow<0.000 \mathrm{I}$ & NA \\
\hline Guideline achievement & & & & & \\
\hline LDL-C $<100$ mg/dL & $38.7 \rightarrow 73.8$ & $39.4 \rightarrow 74.6$ & $37.5 \rightarrow 72.8$ & $<0.01 \rightarrow<0.01$ & $\mathrm{I} .08 \rightarrow \mathrm{I} .10$ \\
\hline $\mathrm{TC}<200 \mathrm{mg} / \mathrm{dL}$ & $66.7 \rightarrow 90.3$ & $66.4 \rightarrow 90.7$ & $67.3 \rightarrow 89.8$ & $0.12 \rightarrow<0.05$ & $0.96 \rightarrow 1.10$ \\
\hline $\begin{array}{l}\mathrm{HDL}-\mathrm{C}>50(\mathrm{w}) / \\
>40 \mathrm{mg} / \mathrm{dL}(\mathrm{m})\end{array}$ & $44.0 \rightarrow 44.4$ & $27.4 \rightarrow 30.8$ & $69.2 \rightarrow 64.9$ & $<0.0001 \rightarrow<0.0001$ & $0.17 \rightarrow 0.24$ \\
\hline $\mathrm{TG}<\mathrm{I} 50 \mathrm{mg} / \mathrm{dL}$ & $58.1 \rightarrow 70.4$ & $43.7 \rightarrow 62.2$ & $80.0 \rightarrow 82.8$ & $<0.000 \mathrm{I} \rightarrow<0.000 \mathrm{I}$ & $0.19 \rightarrow 0.34$ \\
\hline $\begin{array}{l}\mathrm{HDL}-\mathrm{C}>50 / 40 \mathrm{mg} / \mathrm{dL}+ \\
\mathrm{TG}<150 \mathrm{mg} / \mathrm{dL}\end{array}$ & $29.0 \rightarrow 34.6$ & $9.3 \rightarrow 20.7$ & $58.9 \rightarrow 55.6$ & $<0.000 \mathrm{I} \rightarrow<0.000 \mathrm{I}$ & $0.07 \rightarrow 0.21$ \\
\hline $\begin{array}{l}\mathrm{LDL}-\mathrm{C}<100 \mathrm{mg} / \mathrm{dL}+ \\
\mathrm{HDL}-\mathrm{C}>50 \text { or } \\
40 \mathrm{mg} / \mathrm{dL}+\mathrm{TG}<150 \mathrm{mg} / \mathrm{dL}\end{array}$ & $\mathrm{II} .5 \rightarrow 25.9$ & $4.2 \rightarrow 15.9$ & $22.5 \rightarrow 4 I . I$ & $<0.000 \mathrm{I} \rightarrow<0.000 \mathrm{I}$ & $0.15 \rightarrow 0.27$ \\
\hline $\begin{array}{l}\text { Systolic/diastolic BP at entry } \\
\text { and discharge }(\mathrm{mmHg})\end{array}$ & $\begin{array}{l}\mid 30.5 \pm 20.3 / \\
76.5 \pm 11.5 \rightarrow \\
122.3 \pm 14.4 / \\
72.7 \pm 9.3\end{array}$ & $\begin{array}{l}132.8 \pm 20.5 / \\
77.2 \pm 1 \mid .7 \rightarrow \\
123.9 \pm|4.4| \\
73.1 \pm 9.4\end{array}$ & $\begin{array}{l}127.4 \pm 19.6 / \\
75.7 \pm 11.2 \rightarrow \\
120.2 \pm 14.1 / \\
72.2 \pm 9.1\end{array}$ & $\begin{array}{l}<0.000 \mathrm{I} /<0.000 \mathrm{I} \rightarrow \\
<0.000 \mathrm{I} / 0.000 \mathrm{I}\end{array}$ & NA \\
\hline $\mathrm{HbA}_{\mathrm{Ic}}(\%)$ (entry only) & $6.5 \pm 1.1$ & $6.6 \pm 1.1$ & $6.1 \pm 1.0$ & $<0.0001$ & NA \\
\hline Creatinine $(\mathrm{mg} / \mathrm{dL})$ & $\begin{array}{l}\mathrm{I} .2 \pm 0.9 \rightarrow \\
\mathrm{I} .3 \pm 0.95\end{array}$ & $\begin{array}{l}\mathrm{I} .2 \pm 0.9 \rightarrow \\
\mathrm{I} .3 \pm 0.95\end{array}$ & $\begin{array}{l}\text { I. } 2 \pm 0.9 \rightarrow \\
1.2 \pm 0.95\end{array}$ & $<0.000 \mathrm{I} \rightarrow<0.000 \mathrm{I}$ & NA \\
\hline Fasting blood glucose (mg/dL) & $\begin{array}{l}108.1 \pm 33.3 \rightarrow \\
103.3 \pm 27.0\end{array}$ & $\begin{array}{l}115.08 \pm 36.21 \rightarrow \\
108.57 \pm 29.23\end{array}$ & $\begin{array}{l}97.63 \pm 25.01 \rightarrow \\
94.53 \pm 20.03\end{array}$ & $<0.000 \mathrm{I} \rightarrow<0.000 \mathrm{I}$ & NA \\
\hline Max exercise (Watts) & $\begin{array}{l}77.3 \pm 37.0 \rightarrow \\
93.7 \pm 39.3\end{array}$ & $\begin{array}{l}75.5 \pm 36.4 \rightarrow \\
91.1 \pm 38.8\end{array}$ & $\begin{array}{l}79.3 \pm 37.5 \rightarrow \\
96.9 \pm 39.6\end{array}$ & $<0.000 \mathrm{I} \rightarrow<0.000 \mathrm{I}$ & NA \\
\hline
\end{tabular}

Notes: $* P$ values and odds ratios refer to the comparison between the two groups (MetS vs no MetS) at entry and (indicated by the arrow $\rightarrow$ ) at discharge. Abbreviations: MetS, metabolic syndrome; TC, total cholesterol; NA, not applicable; LDL-C, low density lipoprotein cholesterol; HDL-C, high density lipoprotein cholesterol; TG, triglycerides; w, women; m, men; BP, blood pressure; $\mathrm{HbA}_{\mathrm{Ic}}$, glycosylated hemoglobin (HbA $\mathrm{Ic}_{\mathrm{c}}$ values refer to entry visit). 
$\%$

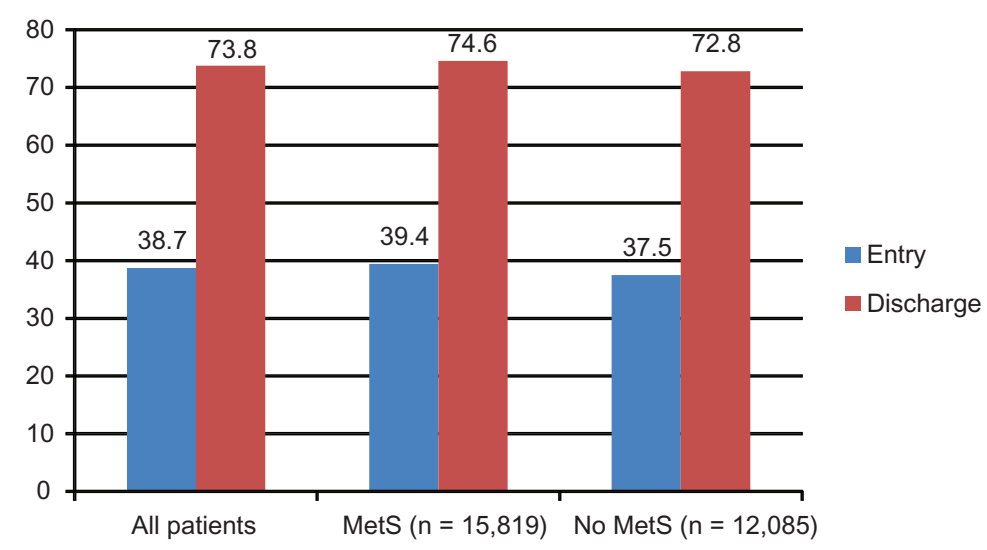

Figure IA LDL-C control rates.

Notes: LDL-C control was defined as $<100 \mathrm{mg} / \mathrm{dL}$.

Abbreviations: LDL-C, low density lipoprotein cholesterol; MetS, metabolic syndrome.

$\%$

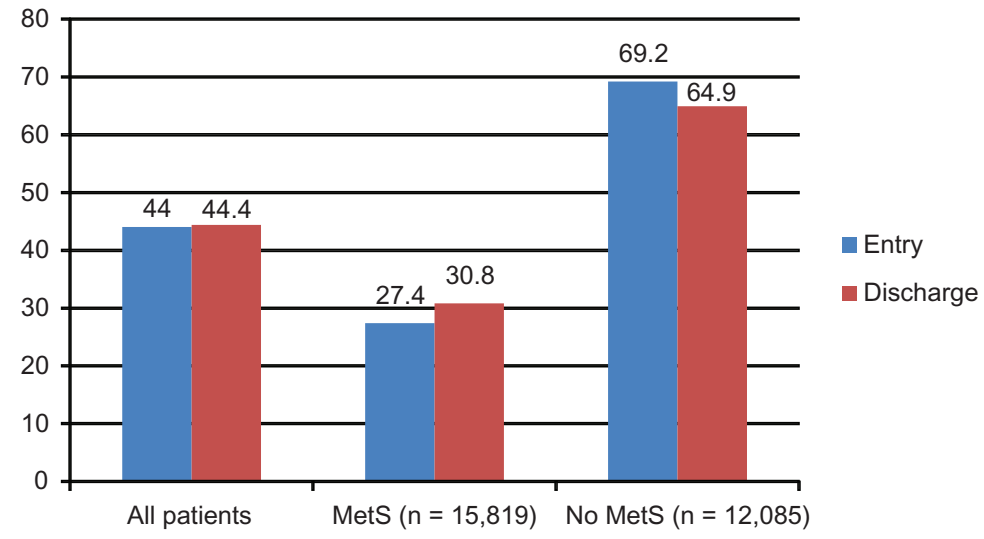

Figure IB HDL-C control rates.

Notes: HDL-C control was defined as $>50 \mathrm{mg} / \mathrm{dL}$ in females and $>40 \mathrm{mg} / \mathrm{dL}$ in males.

Abbreviations: HDL-C, high density lipoprotein cholesterol; MetS, metabolic syndrome.

$\%$

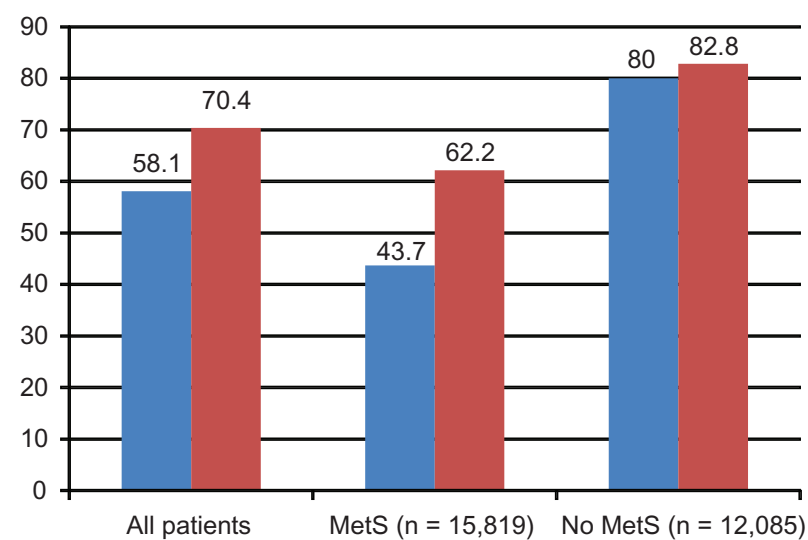

Entry

Discharge

Figure IC TG control rates at entry and discharge.

Notes: TG control was defined as $<150 \mathrm{mg} / \mathrm{dL}$.

Abbreviations: TG, triglycerides; MetS, metabolic syndrome. 
$\%$

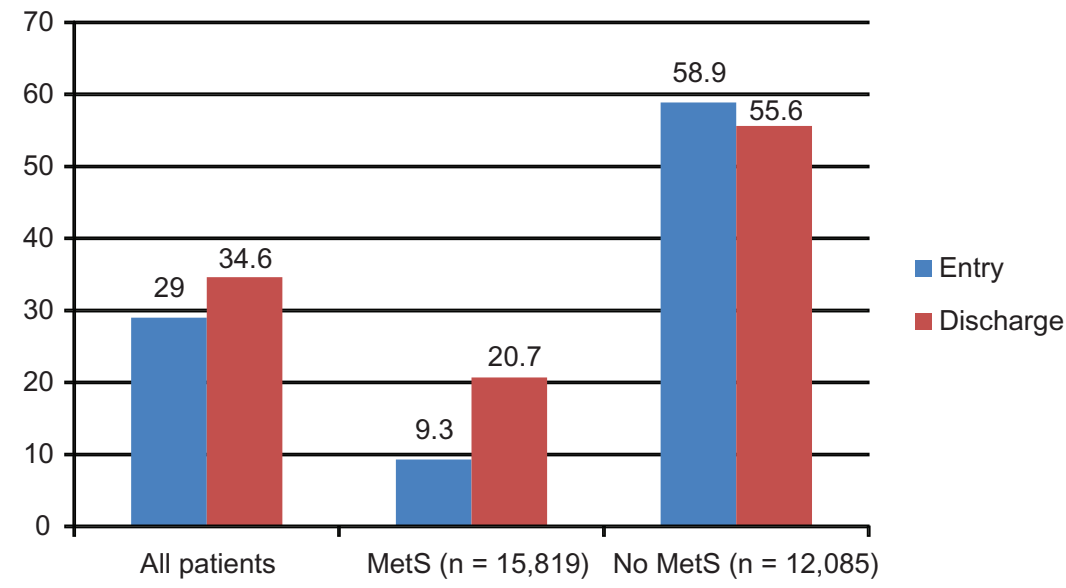

Figure ID Combined control rates (HDL-C and TG).

Notes: HDL-C control was defined as $>50 \mathrm{mg} / \mathrm{dL}$ in females and $>40 \mathrm{mg} / \mathrm{dL}$ in males. TG control was defined as $<150 \mathrm{mg} / \mathrm{dL}$.

Abbreviations: HDL-C, high density lipoprotein cholesterol; TG, triglycerides; MetS, metabolic syndrome.

Mean fasting blood glucose decreased from $108 \mathrm{mg} / \mathrm{dL}$ to $103 \mathrm{mg} / \mathrm{dL}$. $\mathrm{HbA}_{\mathrm{lc}}$ was only available at entry (mean: 6.5\%).

\section{Subgroups with and without MetS}

Mean HDL-C was lower and mean TG were substantially higher in MetS patients compared to those without MetS. Target level attainment rates for LDL-C were somewhat higher for patients with MetS at entry, but much lower for HDL-C and TG (Table 4). This also applied for the combined lipid goals. This pattern did not change at the discharge visit, but overall control rates for all described single parameters (with the exception of HDL-C) had improved substantially.

Mean blood pressure did not differ substantially between groups at discharge, and was in the normal range. Exercise capacity was lower in patients with MetS (91 versus 97 Watts).
Fasting blood glucose (as a component of the MetS definition), was higher in the MetS group and was substantially decreased at discharge. $\mathrm{HbA}_{1 \mathrm{c}}$ was slightly worse in MetS patients compared to those without MetS at entry.

\section{Discussion}

The present analysis provides a snapshot of the characteristics, treatment, and risk factor control of patients with MetS in the CR setting in Germany. More than half of patients had MetS. Irrespective of whether MetS was present or not, patients experienced substantial improvement in particular with respect to lipid parameters, but also physical training.

\section{Patient characteristics}

Patients with or without MetS were similar in terms of gender distribution and age. The latter finding is notable, because in

$\%$

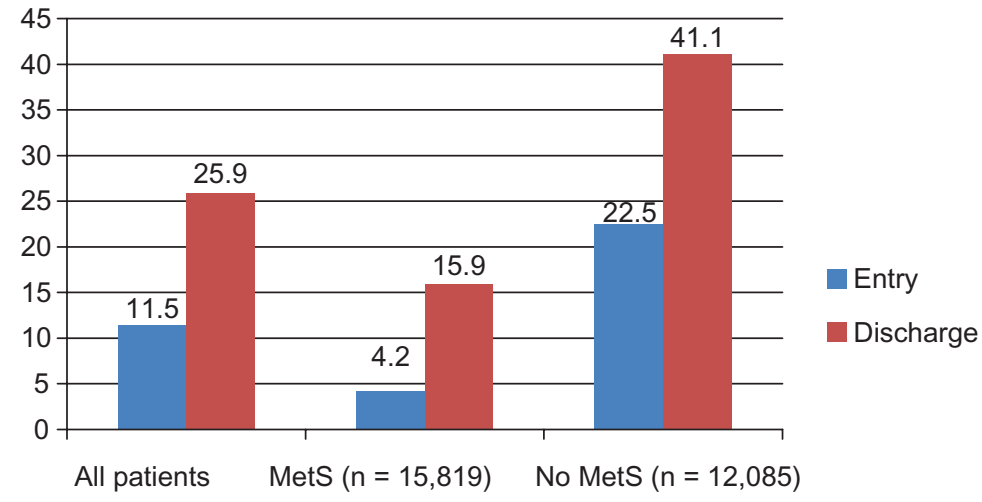

Figure IE Combined control rates (LDL-C, HDL-C, and TG).

Notes: LDL-C control was defined as $<100 \mathrm{mg} / \mathrm{dL}$. HDL-C control was defined as $>50 \mathrm{mg} / \mathrm{dL}$ in females and $>40 \mathrm{mg} / \mathrm{dL}$ in males. TG control was defined as $<\mathrm{I} 50 \mathrm{mg} / \mathrm{dL}$. Abbreviations: LDL-C, low density lipoprotein cholesterol; HDL-C, high density lipoprotein cholesterol; TG, triglycerides; MetS, metabolic syndrome. 
patients with higher body weight myocardial events tend to occur at a younger age (eg, in the CRUSADE registry mean age at first NSTEMI was 75 years in patients with BMI $<18.5 \mathrm{~kg} / \mathrm{m}^{2}$, and 59 years in patients with BMI $\left.>40 \mathrm{~kg} / \mathrm{m}^{2}\right) .{ }^{18}$ Patients with MetS as stipulated by the definition of the syndrome were more often obese, and more often had diabetes mellitus and arterial hypertension. Furthermore, they received more antidiabetic or renin-angiotensin-aldosterone system acting drugs, while the utilization of lipid lowering drugs was similar to patients without MetS. According to our thorough literature search, only one small study has reported in detail the prevalence and profile of MetS in CR following acute coronary syndromes. ${ }^{19}$ In a cohort of 225 patients, age (66.7 years) and gender distribution (men 71\%) was similar to our study, and a similar proportion (58\%) had MetS according to NCEP ATP III criteria, and $65 \%$ were on statins. In that study, 3 months' outpatient treatment reduced the mean number of metabolic derangements significantly (from 3.3 to 2.8), and owing to these changes, the prevalence of MetS decreased to $53 \%$ in the total cohort.

\section{Definition of MetS}

Our analysis was limited to the IDF definition of MetS, and we did not investigate other definitions. Milionis et al have stated in a comparison of three definitions (NCEP ATP III ${ }^{5}$, National Heart, Lung, and Blood Institute/American Heart Association, and IDF), that the latter markedly increased prevalence compared with the other criteria. ${ }^{20}$ In a Greek study representing the overall population, adopting the IDF definition increased the prevalence of MetS substantially compared with NCEP ATP III criteria (IDF 43.4\% versus NCEP $24.5 \%) .{ }^{21}$ As this was a retrospective analysis, physicians were not aware of the MetS condition, unless they diagnosed it themselves. As the value of the MetS diagnosis (beyond its components) compared to simpler risk assessment tools is controversial, ${ }^{22,23-25}$ the effect on the treatment of individual risk factors was independent from this diagnosis.

The target values for lipids, ${ }^{26}$ blood pressure, ${ }^{27}$ or blood glucose ${ }^{28}$ and the medication recommendations for patients with coronary heart disease do not differ in the CR setting ${ }^{29}$ from patients seen by family physicians. In a previous analysis of the dataset, we had noted that drug treatment in CR appears to have been intensified (in terms of drug classes, drugs, and doses) compared to previous years. ${ }^{17}$

An aspect that deserves particular attention is the physical fitness of patients. Previous studies have shown that poor physical fitness is associated with MetS., ${ }^{71,25}$ The combination of both MetS and low fitness (as evidenced by high heart rate $\geq 83$ beats per minute in another study) has very recently been reported to be associated with an almost fourfold increased risk for cardiovascular disease. ${ }^{30}$ In our study, patients in both the MetS and non-MetS groups achieved a considerable gain in exercise capacity (in Watts) during CR which might contribute to improved prognosis in the long term.

\section{Treatment}

Treatment rates with cholesterol absorption inhibitors increased substantially during CR in patients with or without MetS, while statins in terms of drugs and doses were infrequently changed. The leading statin in practice and in the present study was simvastatin, which is usually administered at doses of 20-40 mg/day for coronary heart disease prevention. High simvastatin doses have been associated with increased risk of myopathy, which might discourage physicians to increase doses. ${ }^{31}$ Furthermore, substantial combination data are available for simvastatin/cholesterol absorption inhibitors ${ }^{32}$ and, as in other indications (such as arterial hypertension or chronic heart failure), combination treatment appears adequate in patients who have not achieved their treatment targets on monotherapy.

\section{Control of risk factors}

Despite the relatively short duration of CR stays, a substantial improvement in lipid parameters was achieved. In particular, TG in the MetS group was reduced from 178 to $149 \mathrm{mg} / \mathrm{dL}$, an effect that was much stronger than in the group without MetS. In addition, in the MetS group effects on TC, LDL-C, and HDL-C were stronger than in the group without MetS. Both groups benefited in terms of a notable blood pressure reduction and a reduction of fasting blood glucose at discharge compared to entry. If the lipid control rates as stipulated by the respective guidelines (eg, LDL-C target $<100 \mathrm{mg} / \mathrm{dL}$ ) are taken into account, a substantial improvement after the CR stay was also noted (with the exception of HDL-C). Nonetheless, as in previous studies of patients with coronary heart disease in the primary care setting ${ }^{33,34}$ there is further room for improvement in the lipid values, ${ }^{35,36} \mathrm{HbA}_{1 \mathrm{c}}$, ${ }^{37}$ and blood pressure control rates. ${ }^{38}$ Physicians in rehabilitation have to initiate lifestyle modifications or drug treatment (about 3-4 weeks), and face the complexity of coronary heart disease patients who often have concomitant diseases. ${ }^{39}$

\section{Methodological aspects}

Among the strengths of the registry are its prospective conduct, its large size and representativeness for the German 
CR setting, as well as various quality measures (plausibility checks and others). However, selection bias cannot be excluded for physicians (those voluntarily participating in a registry are more likely to have an interest and probably increased knowledge in the topic) and patients (participants may be more adherent to therapy compared to those declining). In addition, missing data or under-reporting of characteristics may decrease robustness of results. We did not collect data on further potentially informative parameters such as uric acid, pro-coagulation, or inflammatory markers.

\section{Conclusion}

We found that the proportion of patients with MetS was very high among patients who were enrolled in CR programs. Within a short period of, on average, 3-4 weeks, $\mathrm{CR}$ programs led to considerable improvements in patients irrespective of MetS status in terms of mean TC, LDL-C, and TG levels (HDL-C levels also improved in MetS patients). A substantial improvement of risk factors including hypertension and high blood glucose was achieved. Physical fitness that can lower the risk of cardiovascular disease improved in MetS patients to a stronger degree than in patients without MetS. While overall control rates for lipids, blood pressure, and blood glucose are not yet optimal, patients with MetS gain substantial benefit from participation in CR programs.

\section{Acknowledgments}

The TROL registry was conducted in cooperation with the German Society for Prevention and Rehabilitation. It was supported by MSD Sharp and Dohme GmbH, Munich-Haar, Germany. CJ and BK are fulltime employees of MSD. All authors are in the Steering Board or Scientific Board of the study. We acknowledge the input of D Pittrow, Institute for Clinical Pharmacology, Technical University Dresden, for interpretation of the data and various sections of the manuscript.

\section{Disclosure}

The authors declare no conflict of interest related to this work.

\section{References}

1. Greenland P, Alpert JS, Beller GA, et al. 2010 ACCF/AHA guideline for assessment of cardiovascular risk in asymptomatic adults: a Report of the American College of Cardiology Foundation/American Heart Association Task Force on Practice Guidelines. $J$ Am Coll Cardiol. 2010;56(25):e50-e103.

2. World Health Organization. Definition, diagnosis and classification of diabetes mellitus and its complications. Report of a WHO Consultation. Part 1: Diagnosis and classification of diabetes mellitus. Geneva; 1999.
3. Kerner W, Brückel J, Böhm B. Definition, classification and diagnostics of diabetes mellitus. Guidelines of the German Diabetes Society [Deutsche Diabetes Gesellschaft DDG]. Scherbaum WA, Kiess W, eds. 2004. German.

4. Einhorn D, Reaven GM, Cobin RH, et al. American College of Endocrinology position statement on the insulin resistance syndrome. Endocr Pract. 2003;9(3):237-252.

5. Grundy SM, Brewer HB Jr, Cleeman JI, et al. Definition of metabolic syndrome: Report of the National Heart, Lung, and Blood Institute/ American Heart Association conference on scientific issues related to definition. Circulation. 2004;109(3):433-438.

6. Bloomgarden ZT. American Association of Clinical Endocrinologists (AACE) consensus conference on the insulin resistance syndrome: August 25-26, 2002, Washington, DC. Diabetes Care. 2003;26(3): 933-939.

7. Internation Diabetes Federation. IDF worldwide definition of the metabolic syndrome. Available from: http://www.idf.org/idfworldwide-definition-metabolic-syndrome. Accessed on December 22, 2010.

8. Ades PA. Cardiac rehabilitation and secondary prevention of coronary heart disease. N Engl J Med. 2001;345(12):892-902.

9. Karoff M, Held K, Bjarnason-Wehrens B. Cardiac rehabilitation in Germany. Eur J Cardiovasc Prev Rehabil. 2007;14(1):18-27.

10. Haskell WL, Alderman EL, Fair JM, et al. Effects of intensive multiple risk factor reduction on coronary atherosclerosis and clinical cardiac events in men and women with coronary artery disease. The Stanford Coronary Risk Intervention Project (SCRIP). Circulation. 1994;89(3): 975-990.

11. Niebauer J, Hambrecht R, Velich T, et al. Attenuated progression of coronary artery disease after 6 years of multifactorial risk intervention: role of physical exercise. Circulation. 1997;96(8):2534-2541.

12. Ornish D, Scherwitz LW, Billings JH, et al. Intensive lifestyle changes for reversal of coronary heart disease. JAMA. 1998;280(23):2001-2007.

13. O'Connor GT, Buring JE, Yusuf S, et al. An overview of randomized trials of rehabilitation with exercise after myocardial infarction. Circulation. 1989;80(2):234-244.

14. Willich SN, Muller-Nordhorn J, Kulig M, et al. Cardiac risk factors, medication, and recurrent clinical events after acute coronary disease; a prospective cohort study. Eur Heart J. 2001;22(4):307-313.

15. Bestehorn K, Wegscheider K, Voller H. Contemporary trends in cardiac rehabilitation in Germany: patient characteristics, drug treatment, and risk-factor management from 2000 to 2005. Eur J Cardiovasc Prev Rehabil. 2008;15(3):312-318.

16. German Society for Prevention and Rehabilitation of Cardiovascular Disease [Deutsche Gesellschaft für Prävention und Rehabilitation von Herz-Kreislauferkrankungen e.V., Koblenz, Germany]. Available from: http://www.dgpr.de. Accessed March 28, 2012. German.

17. Völler H, Reibis R, Pittrow D, et al. Secondary prevention of diabetic patients with coronary artery disease in cardiac rehabilitation: risk factors, treatment and target level attainment. Curr Med Res Opin. 2009;25(4):879-890.

18. Madala MC, Franklin BA, Chen AY, et al. Obesity and age of first non-ST-segment elevation myocardial infarction. J Am Coll Cardiol. 2008;52(12):979-985.

19. Milani RV, Lavie CJ. Prevalence and profile of metabolic syndrome in patients following acute coronary events and effects of therapeutic lifestyle change with cardiac rehabilitation. Am J Cardiol. 2003;92(1):50-54.

20. Milionis HJ, Kostapanos MS, Liberopoulos EN, et al. Different definitions of the metabolic syndrome and risk of first-ever acute ischaemic non-embolic stroke in elderly subjects. Int J Clin Pract. 2007;61(4):545-551.

21. Athyros VG, Ganotakis ES, Elisaf M, Mikhailidis DP. The prevalence of the metabolic syndrome using the National Cholesterol Educational Program and International Diabetes Federation definitions. Curr Med Res Opin. 2005;21(8):1157-1159. 
22. Kahn R, Buse J, Ferrannini E, Stern M. The metabolic syndrome: time for a critical appraisal: joint statement from the American Diabetes Association and the European Association for the Study of Diabetes. Diabetes Care. 2005;28(9):2289-2304.

23. Kahn R. Metabolic syndrome - what is the clinical usefulness? Lancet. 2008;371(9628):1892-1893.

24. Sattar N, McConnachie A, Shaper AG, et al. Can metabolic syndrome usefully predict cardiovascular disease and diabetes? Outcome data from two prospective studies. Lancet. 2008;371(9628):1927-1935.

25. Grundy SM. Does a diagnosis of metabolic syndrome have value in clinical practice? Am J Clin Nutr. 2006;83(6):1248-1251.

26. Expert Panel on Detection, Evaluation, and Treatment of High Blood Cholesterol in Adults. Executive Summary of the Third Report of the National Cholesterol Education Program (NCEP) Expert Panel on Detection, Evaluation, and Treatment of High Blood Cholesterol in Adults (Adult Treatment Panel III). JAMA. 2001;285(19): 2486-2497.

27. Chobanian AV, Bakris GL, Black HR, et al. The seventh report of the Joint National Committee on Prevention, Detection, Evaluation, and Treatment of High Blood Pressure: the JNC 7 report. JAMA. 2003; 289(19):2560-2571.

28. Ryden L, Standl E, Bartnik M, et al. Guidelines on diabetes, prediabetes, and cardiovascular diseases: executive summary: The Task Force on Diabetes and Cardiovascular Diseases of the European Society of Cardiology (ESC) and of the European Association for the Study of Diabetes (EASD). Eur Heart J. 2007;28(1):88-136.

29. Balady GJ, Ades PA, Comoss P, et al. Core components of cardiac rehabilitation/secondary prevention programs: a statement for healthcare professionals from the American Heart Association and the American Association of Cardiovascular and Pulmonary Rehabilitation Writing Group. Circulation. 2000;102(9):1069-1073.

30. O'Hartaigh B, Jiang CQ, Thomas GN, et al. Usefulness of physical fitness and the metabolic syndrome to predict vascular disease risk in older Chinese (from the Guangzhou Biobank Cohort Study-Cardiovascular Disease Subcohort [GBCS-CVD]). Am J Cardiol. 2011;108(6): $845-850$.
31. Food and Drug Administration(FDA). FDA Drug Safety Communication: New restrictions, contraindications, and dose limitations for Zocor (simvastatin) to reduce the risk of muscle injury.Available from: http://www.fda.gov/Drugs/DrugSafety/ucm256581.htm. Accessed March 27, 2012.

32. Ijioma N, Robinson JG. Lipid-lowering effects of ezetimibe and simvastatin in combination. Expert Rev Cardiovasc Ther. 2011;9(2): 131-145.

33. Bestehorn K, Jannowitz C, Karmann B, Pittrow D, Kirch W. Characteristics, management and attainment of lipid target levels in patients enrolled in Disease Management Program versus those in routine care: LUTZ registry. BMC Public Health. 2009;9(1):280.

34. Gitt AK, Juenger C, Jannowitz C, Karmann B, Senges J, Bestehorn K. Guideline-oriented ambulatory lipid-lowering therapy of patients at high risk for cardiovascular events by cardiologists in clinical practice: the 2L cardio registry. Eur J Cardiovasc Prev Rehabil. 2009;16(4): 438-444.

35. Gitt AK, Junger C, Smolka W, Bestehorn K. Prevalence and overlap of different lipid abnormalities in statin-treated patients at high cardiovascular risk in clinical practice in Germany. Clin Res Cardiol. 2010;99(11):723-733.

36. Böhler S, Scharnagl H, Freisinger F, et al. Unmet needs in the diagnosis and treatment of dyslipidemia in the primary care setting in Germany. Atherosclerosis. 2007;190(2):397.

37. Pittrow D, Stalla GK, Zeiher AM, et al. Prevalence, drug treatment and metabolic control of diabetes mellitus in primary care. Med Klin (Munich). 2006;101(8):635-644.

38. Sharma A, Wittchen H, Kirch W, et al. High prevalence and poor control of hypertension in primary care: cross sectional study. $J$ Hypertens. 2004;22:479-486.

39. Pittrow D, Kirch W, Bramlage P, et al. Patterns of antihypertensive drug utilization in primary care. Eur J Clin Pharmacol. 2004;60(2): 135-142.

40. Magliano DJ, Shaw JE, Zimmet PZ. How to best define the metabolic syndrome. Ann Med. 2006;38(1):34-41.
Vascular Health and Risk Management

\section{Publish your work in this journal}

Vascular Health and Risk Management is an international, peerreviewed journal of therapeutics and risk management, focusing on concise rapid reporting of clinical studies on the processes involved in the maintenance of vascular health; the monitoring, prevention and treatment of vascular disease and its sequelae; and the involvement of

\section{Dovepress}

metabolic disorders, particularly diabetes. This journal is indexed on PubMed Central and MedLine. The manuscript management system is completely online and includes a very quick and fair peer-review system, which is all easy to use. Visit http://www.dovepress.com/ testimonials.php to read real quotes from published authors. 